\title{
Anhang D: Korrespondenzen des Clubs ${ }^{1}$
}

\begin{tabular}{|c|c|}
\hline Club & Département \\
\hline Paris & Seine (75) \\
\hline Marseille & Bouches-du-Rhône (13) \\
\hline Wissembourg & Bas-Rhin (67) \\
\hline Barr & Bas-Rhin (67) \\
\hline Colmar & Haut-Rhin (68) \\
\hline Huningue & Haut-Rhin (68) \\
\hline Lille & Nord (59) \\
\hline Nancy & Meurthe (54) \\
\hline Sélestat & Bas-Rhin (67) \\
\hline Lauterbourg & Bas-Rhin (67) \\
\hline Neuf-Brisach & Haut-Rhin (68) \\
\hline Beaune & Côte-d'Or (21) \\
\hline Bordeaux & Gironde (33) \\
\hline Saint-Dié & Vosges (88) \\
\hline Wasselonne & Bas-Rhin (67) \\
\hline Aix-en-Provence & Bouches-du-Rhône (13) \\
\hline Bar-le-Duc & Meuse (55) \\
\hline Besançon & Doubs (25) \\
\hline Bischwiller & Bas-Rhin (67) \\
\hline Blamont & Meurthe (54) \\
\hline Chalon-sur-Saône & Saône-et-Loire (71) \\
\hline Dijon & Côte-d'Or (21) \\
\hline Limoges & Haute-Vienne (87) \\
\hline Niort & Deux-Sèvres (79) \\
\hline Saint-Avold & Moselle (57) \\
\hline Saverne & Bas-Rhin (67) \\
\hline Ambert & Puy-de-Dôme (63) \\
\hline Bayonne & Basses-Pyrénées (64) \\
\hline Belfort & Haut-Rhin (68) \\
\hline Brignoles & $\operatorname{Var}(83)$ \\
\hline Cernay & Haut-Rhin (68) \\
\hline Chambéry & Mont-Blanc (73) \\
\hline Delle & Haut-Rhin (68) \\
\hline Epinal & Vosges $(88)$ \\
\hline
\end{tabular}

' Diese Aufstellung wertet sämtliche erhaltenen Korrespondenzen des Straßburger Clubs mit anderen Gesellschaften aus. Sie entstand auf der Grundlage des Findbuches SCHWICKER, Inventaire analytique. 


\begin{tabular}{|c|c|}
\hline Gray & Haute-Saône (70) \\
\hline Grenoble & Isère (38) \\
\hline Josselin & Morbihan (56) \\
\hline Landau & Bas-Rhin (67) \\
\hline Lisieux & Calvados (14) \\
\hline Lorient & Morbihan (56) \\
\hline Moyenvic & Moselle (57) \\
\hline Nantes & Loire-Inférieure (44) \\
\hline Neuviller-la-Roche & Bas-Rhin (67) \\
\hline Périgeux & Dordogne (24) \\
\hline Perpignan & Pyrénées-Orientales (66) \\
\hline Phalsbourg & Moselle (57) \\
\hline Remiremont & Vosges $(88)$ \\
\hline Saint-Amour & Jura (39) \\
\hline Sainte-Marie-aux-Mines & Haut-Rhin (68) \\
\hline Sedan & Ardennes (08) \\
\hline Tours & Indre-et-Loire (37) \\
\hline Tulle & Correze (19) \\
\hline Ajaccio & Liamone (20) \\
\hline Allevard & Isère (38) \\
\hline Andlau & Bas-Rhin (67) \\
\hline Argentat & Corrèze (19) \\
\hline Arnay-le-Duc & Côte-d'Or (21) \\
\hline Arpajon & Cantal (15) \\
\hline Auxerre & Yonne (89) \\
\hline Avignon & Vaucluse (84) \\
\hline Bayeux & Calvados (14) \\
\hline Belvès & Dordogne (24) \\
\hline Benfeld & Bas-Rhin (67) \\
\hline Bergerac & Dordogne (24) \\
\hline Béziers & Hérault (34) \\
\hline Blaye & Gironde (33) \\
\hline Blois & Loir-et-Cher (41) \\
\hline Bourg-en-Bresse & Ain $(01)$ \\
\hline Calais & Pas-de-Calais (62) \\
\hline Chalons-sur-Marne & Marne (51) \\
\hline Charolles & Saône-et-Loire (71) \\
\hline Chateau-du-Loir & Sarthe (72) \\
\hline Dax & Landes (40) \\
\hline Dieuze & Moselle (57) \\
\hline Dole & Jura (39) \\
\hline Dunkerque & Nord (59) \\
\hline
\end{tabular}




\begin{tabular}{|c|c|}
\hline Ebersmunster & Bas-Rhin (67) \\
\hline Fort-Louis & Bas-Rhin (67) \\
\hline Giromagny & Haut-Rhin (68) \\
\hline Hindisheim & Bas-Rhin (67) \\
\hline L'Isle-sur-le-Doubs & Doubs (25) \\
\hline La Petite Pierre & Bas-Rhin (67) \\
\hline La Rochelle & Charente-Inférieure (17) \\
\hline La-Mothe-Saint-Héraye & Deux-Sèvres (79) \\
\hline Le Mans & Sarthe (72) \\
\hline Ligny-en-Barrois & Meuse (55) \\
\hline Limoux & Aude (11) \\
\hline Londres & England \\
\hline Lons-le-Saunier & Jura (39) \\
\hline Louhans & Saône-et-Loire (71) \\
\hline Lyon & Rhône (69) \\
\hline Mâcon & Saône-et-Loire (71) \\
\hline Marckolsheim & Bas-Rhin (67) \\
\hline Marennes & Charente-Inférieure (17) \\
\hline Melun & Seine-et-Marne (77) \\
\hline Mende & Lozère (48) \\
\hline Montauban & Lot (46) \\
\hline Mouzon & Ardennes (08) \\
\hline Moyaux & Calvados (14) \\
\hline Nice & Alpes-Maritimes (06) \\
\hline Orléans & Loiret (45) \\
\hline Paimbøuf & Loire-Inférieure (44) \\
\hline Poitiers & Vienne (86) \\
\hline Pontarlier & Doubs (25) \\
\hline Pontoise & Seine-et-Oise (78) \\
\hline Porrentruy & Mont-Terrible (90) \\
\hline Rennes & Ille-et-Vilaine (35) \\
\hline Rochefort & Charente- Inférieure (17) \\
\hline Rosheim & Bas-Rhin (67) \\
\hline Rothau & Bas-Rhin (67) \\
\hline Rouen & Seine-Inférieure (76) \\
\hline Saint-Denis & Seine $(75)$ \\
\hline Saint-Jean-de-Bournay & Isère (38) \\
\hline Saint-Jean-de-Losne & Côte-d'Or (21) \\
\hline Saint-Jean-du-Gard & Gard (30) \\
\hline Saint-Loup & Haute-Saône (70) \\
\hline Saint-Omer & Pas-de-Calais (62) \\
\hline Sarrelouis & Moselle (57) \\
\hline
\end{tabular}


Sarre-Union

Tarascon

Thann

Tonneins

Toul

Toulon

Toulouse

Troyes

Ussel

Verdun

Verdun-sur-le-Doubs

Versailles

Vesoul

Waldersbach
Bas-Rhin (67) 1

Bouches-du-Rhône (13) 1

Haut-Rhin (68) 1

Lot-et-Garonne (47) 1

Meurthe (54) 1

$\operatorname{Var}(83)$

Haute-Garonne (31) 1

Aube (10) 1

Corrèze (19) 1

Meuse (55) 1

Saône-et-Loire (71) 1

Seine-et-Oise (78) 1

Haute-Saône (70) 1

Bas-Rhin (67) 1 
Korrespondenzen des Clubs pro Département

Département

67 Bas-Rhin

68 Haut-Rhin

75 Seine

13 Bouches-du-Rhône

54 Meurthe

21 Côte-d'Or

57 Moselle

88 Vosges

59 Nord

71 Saône-et-Loire

25 Doubs

33 Gironde

55 Meuse

14 Calvados

19 Corrèze

24 Dordogne

38 Isère

39 Jura

56 Morbihan

70 Haute-Saône

79 Deux-Sèvres

8 Ardennes

17 Charente-Inférieure

44 Loire-Inférieure

83 Var

87 Haute-Vienne

37 Indre-et-Loire

62 Pas-de-Calais

63 Puy-de-Dôme

64 Basses-Pyrénées

66 Pyrénées-Orientales

72 Sarthe

73 Mont-Blanc

78 Seine-et-Oise

1 Ain

6 Alpes-Maritimes

10 Aube

11 Aude
Anzahl der Briefe

49

27

12

11

10

9

9

8

7

7

5

5

5

4

4

4

4

4

4

4

4

3

3

3

3

3

2

2

2

2

2

2

2

2

1

1

1 
15 Cantal

20 Liamone

30 Gard

31 Haut-Garonne

34 Hérault

35 Ille-et-Vilaine

40 Landes

41 Loir-et-Cher

45 Loiret

46 Lot

47 Lot-et-Garonne

48 Lozère

51 Marne

69 Rhône

76 Seine-Inférieure

77 Seine-et-Marne

84 Vaucluse

86 Vienne

89 Yonne

90 Mont-Terrible 